\title{
Observation of Serum Electrolytes and Nutritional Status in Patients With Gastric Outlet Obstruction Both Pre and Post Operative Status
}

\author{
Authors \\ Dr Sanjay Kujur ${ }^{1}$, Dr Jitendra Kumar Sahu ${ }^{2}$, Dr Achuytananda Behera ${ }^{3}$ \\ Department of Surgery, VSSIMSAR, Burla, Odisha
}

\section{Introduction}

Gastric outlet obstruction which was previously known as pyloric stenosis is not a single entity, it is the clinical and path physiological consequence of any disease process that produces a mechanical impediment to gastric emptying. Gastric outlet obstruction implies complete or incomplete obstruction of distal stomach, pylorus or proximal duodenum. This may occur as an obstructing mass lesion, external compression or as result of obstruction from acute edema, chronic scarring or fibrosis or combination of both.

Due to continuous loss of gastric juice during vomiting a gross degree of electrolyte imbalance is expected. These patients with gastric outlet obstruction are also malnourished because nutrients are deprived of their exposure to main digestive and absorptive areas as very less amount of nutrients pass beyond pylorus. These patients frequently avoid taking food as there is unpleasant fullness of stomach after taking food.

Morden surgical practice has gained significance not only for growing range and magnitude of operations performed but also reducing morbidity and mortality by management of electrolyte imbalance and improvement of nutritional status.
Sodium is the back bone of extracellular fluid. Its function is to conserve the isotonic balance and thus maintain the volume of fluid in the interstitial space. Excess loss of gastro intestinal secretion id the commonest cause of sodium depletion in gastric obstruction that leads to dehydration Potassium is the predominant intracellular cat ion, its vital function being modernization contraction of heart muscle. There is no adequate mechanism to conserve potassium. Hence loss of potassium is continuous in spite of no intake. Potassium loss is frequently met with loss of gastro intestinal secretion as vomiting.

Malnutrition has long been recognised as a potential cause of mortality and morbidity in surgical patients. In 1936 Studley et al reviewed the operative morbidity and mortality of patients undergoing elective surgery for peptic ulcer disease. he found significant morbidity and mortality if weight loss of more $20 \%$ has occured prior to operative procedure .James et al in 1979 also reported that substantial malnutrition exists in elective surgical patients and correlates with subsequent morbidity and mortality.

Recently data's have been generated identifying specific measures of nutritional status as predictor of increased hospital morbidity and mortality 
secondary to protein calorie malnutrition. Anionio at al in 1992 suggest that when parentral nutrition is given to malnourished surgical patients in adequate amount for 7 to 15 days pre operatively significant improvement in both nutritional status and post operative clinical outcome are likely to occur.

Various authors have disclosed a tree fold increase in the infection of clean wounds in patients with recent weight loss or low serum albumin concentration. Protein calorie malnutrition has been shown to be associated with delayed wound healing, decreased strength of intestinal anastomosis and abdominal wound strength, impaired mature collagen formation and decreased resistance to infection.

When a patient is admitted with gastric out let obstruction, he is dehydrated with hypokalamic, hypochloremic, alkalosis, anaemia, hypoproteinemia and malnourished, ,. To a surgeon it is really a difficult situation to access the feasibility of an operation at first hand in such clinically ill, dehydrated and emaciated patient with severe electrolyte imbalance and marked malnutrition.

Incidence of gastric outlet obstruction is quite high in this region of state. keeping in view of importance of the subject and effect $s$ of electrolyte imbalance and nutritional deficiency, the present study have been undertaken to evaluate the serum electrolyte changes and nutritional assessment in patient $\mathrm{s}$ with gastric outlet obstruction both pre and post operative state .Knowledge of it will be a help for better management of patients.

\section{Aims And Objectives}

The present study is designed and to be carried out to estimate the serum electrolytes and nutritional status in the patients with gastric outlet obstruction both pre and post operatively and analyze the results for the better management of the patients. The aetiology, symptoms, signs, age and gender distribution, operative procedures, postoperative complications and their relationship to various preoperative parameters are to be compared.

\section{Materials And Methods}

Sixty six cases of gastric out let obstruction admitted to the department of surgery VSS Institute of Medical Science And Research, Sambalpur from November 2014 to November 2016 were included in the study. Patients with gastro paresis without mechanical obstruction were excluded from the study,.

The provisional diagnosis was based on detailed clinical history, thorough physical examination , routine laboratory investigation and special investigations like upper GI endoscopy . serum sodium , potassium ., height , weight ,serum albumin were recorded in all cases of provisional diagnosis of gastric outlet obstruction .all patients were managed pre operatively with gastric lavage , fluid and electrolyte replacement , and whenever needed with whole blood /. Pre operative findings operative procedure and post operative complications were noted. On $3^{\text {rd }}$ and $8^{\text {th }}$ post operative day serum sodium, potassium, albumin, and weight were recorded.

\section{Observation}

Sixty six cases of gastric outlet obstruction admitted to the VSS Institute of Medical Science And Research, Burla, Sambalpur from November 2014 to November 2016 were taken in this study. Observation on clinical presentation, diagnosis, operative procedure and complications were recorded and shown in the following tables. Pre and post operative values of serum Sodium and serum Potassium, weight and serum albumin level were recorded and shown in the following tables.

Age incidence is maximum $(33.33 \%)$ in the $6^{\text {th }}$ decade of life with most of the patients belonged to the age group $40-60$ year of age, with a male to female ratio 5.6: 1

Maximum cases were from low socioeconomic group (60.6\%).Incidence of gastric outlet obstruction was more among non vegetarians (90.91\%). 
Non bilious vomiting was found to be the commonest symptom (96.96\%) followed by upper abdomen pain (90.90\%). Loss of appetite $(75.75 \%)$, fullness in the upper abdomen $(68.18 \%)$, weight loss $(72.72 \%)$ were other common symptoms.

Commonest_cause of gastric outlet obstruction was found to be carcinoma of pylorus and antrum $(60.60 \%)$ followed by cicatricial duodenal ulcer $(25.76 \%)$, carcinoma pancreas $(9.1 \%)$, gastric tuberculosis $(1.51 \%)$ and chemical ingestion $(1.51 \%)$.

Carcinomas of gastric antrum 10 cases $(25 \%)$ were treated by partial gastrectomy, and the rest 30 cases $(75 \%)$ were managed by palliative gastrojejunostomy. Truncal vagotomy and gastrojejunostomy was performed in 9 cases (52.9\%) and only gastrojejunostomy was done in 8 cases $(47.1 \%)$ of cicatricial duodenal ulcer. All patients of carcinoma of pancreas (7 cases) were managed by palliative gastrojejunostomy. One patient of GOO due to chemical ingestion was managed by gastrojejunostomy and one patient of gastric tuberculosis was managed by gastrojejunostomy followed by post operative ATT.

In the present study 17 patients $(25.75 \%)$ had 25 post operative complications. The commonest complication being surgical site infection $(32 \%)$ followed by post operative pyrexia (24\%), pneumonia (16\%), diarrhoea (12\%) paralytic ileus $(8 \%)$ and stomal obstruction $(8 \%)$

Serum Sodium concentration in patients with GOO, pre and post operative

\begin{tabular}{|c|c|c|c|c|c|c|}
\hline \multirow{2}{*}{$\begin{array}{l}\text { Serum } \\
\text { Sodium conc. } \\
\text { In Mmol/liter }\end{array}$} & \multicolumn{3}{|l|}{ Preoperative } & \multicolumn{3}{|c|}{ Post operative } \\
\hline & No. of cases & Percentage & Mean & No. of cases & Percentage & Mean \\
\hline $135-145$ & 51 & 77.27 & 136.16 & 66 & 100 & 136.65 \\
\hline $130-134.9$ & 15 & 22.73 & 132.13 & 0 & 0 & 0 \\
\hline
\end{tabular}

The present study shows pre operative fall in sodium level in $22.73 \%$ of cases with a mean of $132.13 \mathrm{mmol} / \mathrm{l}$. During the post operative period all the patients had normal sodium concentration with a mean of $136.66 \mathrm{mmol} / \mathrm{l}$.

Serum Potassium concentration in patients with GOO, pre and post operative

\begin{tabular}{|c|c|c|c|c|c|c|}
\hline \multirow{2}{*}{$\begin{array}{l}\text { Serum Potassium } \\
\text { conc. } \\
\text { In Mmol/liter }\end{array}$} & \multicolumn{3}{|l|}{ Preoperative } & \multicolumn{3}{|c|}{ Post operative } \\
\hline & No. of cases & Percentage & Mean & No. of cases & Percentage & Mean \\
\hline $3.5-5.5$ & 44 & 66.67 & 3.88 & 66 & 100 & 4.2 \\
\hline$<3.5$ & 22 & 33.33 & 2.77 & 0 & 0 & 0 \\
\hline
\end{tabular}

In the present series $33.33 \%$ of cases were operatively the mean weight decreases to $50.88 \mathrm{~kg}$. hypokalemic with a mean potassium level of 2.77 $\mathrm{mmol} / \mathrm{l}$ prior to surgery. Postoperatively all the patients had normal potassium concentration with a mean of $4.2 \mathrm{mmol} / \mathrm{l}$.

$48.48 \%$ patients were under weight with the mean weight of $50.92 \mathrm{~kg}$ during admission. Post

25 complications occurred in 17 patients, of those only 3 complications occurred in those patients with normal weight whereas 22 complications occurred in those with low weight for height at the time of admission

Serum Albumin concentration in patients with GOO, pre and post operative

\begin{tabular}{|c|c|c|c|c|c|c|c|}
\hline \multirow{2}{*}{$\begin{array}{l}\text { Serum } \\
\text { conc. } \\
\text { percenta }\end{array}$} & \multirow{2}{*}{$\begin{array}{l}\text { Albumin } \\
\text { In gm } \\
\text { ge }\end{array}$} & \multicolumn{3}{|l|}{ Preoperative } & \multicolumn{3}{|c|}{ Post operative } \\
\hline & & No. of cases & Percentage & Mean & No. of cases & Percentage & Mean \\
\hline$\geq 3.5$ & & 50 & 75.76 & 3.58 & 47 & 71.21 & 3.50 \\
\hline$<3.5$ & & 16 & 24.24 & & 19 & 28.79 & \\
\hline
\end{tabular}


Serum albumin levels were low in $24.24 \%$ cases as compared to $75.76 \%$ with normal serum albumin level. The mean serum albumin in the pre operative patients was $3.58 \mathrm{gm} \%$.

The total number of postoperative patients with low serum albumin was $28.79 \%$ and the mean serum albumin level in these patients was 3.5 gm\%. Post operatively $56.06 \%$ patients had decreased albumin level as compared to the pre operative level, whereas $6.06 \%$ patients had increased and $37.88 \%$ patients had unchanged serum albumin level. 9 out of 16 patients with hypoalbuminemia $(56.25 \%)$ had some sort of post operative complications, whereas 8 out of 42 patients with normal serum albumin level $(19.04 \%)$ had postoperative complications.

\section{Discussion}

Sixty six patients of gastric outlet obstruction admitted to department of Surgery of VSS Institute of Medical Science And Research, Burla during the period of November 2014 to November 2016 were analysed and discussed now.

Majority of patients in this study belonged to sixth decade of life followed by fifth decade. In malignant cases maximum incidence seen in the older age group as compared to the benign cases, as observed by Balint and Spence (1959), Tydd and Dryer (1974), Kaushik (1976), Hausen (1984),Nayak (1984), Kshitiz(2013), Hyasinta (2013)

$84.84 \%$ of patients are male and $15.16 \%$ are female with a male to female ratio of 5.6:1.which is slightly higher than many studies of Balint and Spance( 1959), Cooke(1977), Nayak(1984), Kshitiz(2013), Hyasinta (2013). But the ratio is nearly equal to the studies of Kaushik and Moses (1973), Yogiram and Chaudhary(1983).The higher incidence in males, worldwide can be explained as because of more consumption of gastric irritant by males compared to females.

$60.6 \%$ low socioeconomic group and $(90.91 \%)$ non vegetarian cases which are similar to studies of Singh (1979), Kshitiz (2013), Hyasinta (2013). Commonest complaint was copious projectile vomiting (96.96\%) followed by pain in the upper abdomen $(90.9 \%)$. Loss of appetite $(75.75 \%)$, fullness in the upper abdomen (68.18\%), weight loss $(72.72 \%)$, something moving in the abdomen (53\%), constipation (36\%), melena (30\%) were the other symptoms. Balint and Spence (1959) reported vomiting in $97.4 \%$, pain abdomen in $88 \%$, and anorexia in $61.8 \%$, weight loss in $65.2 \%$ and diarrhoea in $19.4 \%$ cases with gastric outlet obstruction.Goldstein et al (1966) observed vomiting was present in $83 \%$ cases and pain abdomen in $80 \%$ cases. Kaushik and Moses (1973) found $86.4 \%$ cases had vomiting and $80.2 \%$ had pain abdomen and $28.2 \%$ had weight loss in duodenal ulcer stenosis. Wiland et al (1982) reported $91 \%$ cases had vomiting, $86 \%$ had pain abdomen, 52\% had weight loss. Nayak (1984) observed vomiting in $100 \%$ cases, pain abdomen in $80 \%$ cases and anorexia $56 \%$ cases of gastric outlet obstruction due to gastric carcinoma. Jaka et al (2013) found non bilious vomiting being the most consistent symptom present in $100 \%$ of cases followed by weight loss (93.5) case.

Ranka Kshitiz(2013) found, vomiting(100\%) as the most common and constant symptom and G.O.O. Other symptoms are pain abdomen, loss of appetite, loss of weight, post prandial fullness, malena.

In the present study vomiting is the most common symptom followed by pain in upper abdomen in gastric outlet obstruction irrespective of the cause and is similar to the findings of Balint and Spence (1959), Wiland et al (1982), Goldstein et al (1966), Jaka et al (2013), Ranka Kshitiz(2013). In the present study loss of appetite is found in $75.7 \%$ of cases which is in accordance with the findings of Ranka Kshitiz(2013), Nayak (1984), Balint and Spence (1959). Weight loss is present in $72.7 \%$ cases and post prandial fullness is found in $68 \%$ case which is in accordance with the findings of Jaka et al (2013), Ranka Kshitiz(2013). Constipation found in $36.3 \%$ cases is similar to the study of Kaushik and Moses (1973).malena found in $30 \%$ cases is similar to the study of Ranka Kshitiz(2013).

Dehydration is found in $30.3 \%$ of cases which is in consistent with the study of Jaka et al (2013). 
The commonest cause of gastric outlet obstruction is found to be carcinoma of gastric antrum $(60.60 \%)$ followed by cicatricial duodenal ulcer $(25.76 \%)$ these observations are similar to that of Ranka Kshitiz(2013), Jaka et al (2013), Abdul samad (2007), Dallas N Shone et al (2010), Mc Quaid Kenneth et al (2010), Derek Frederickson (2008).

These findings are in contrast to the findings of Balint and Spence (1959), Goldstein et al (1966), Kreel Ellis (1965), Kotisso R (2000). All these authors found benign gastric outlet obstruction (peptic ulcer disease) as the leading cause of gastric outlet obstruction.

Until late 1970, Benign diseases were the major cause of G.O.O and only 10-39\% cases of G.O.O were because of malignancy but after that due to widespread use of proton pump inhibitors \& $\mathrm{H}$. pylori eradication, predominant causes of G.O.O. have changed substantively, now malignancy accounts for $50-80 \%$ cases of G.O.O.

The other cause of GOO in the present study includes carcinoma of pancreas $(9.1 \%)$, gastric tuberculosis (1.5\%) and chemical ingestion (1.5\%).

Out of 40 cases of carcinoma of gastric antrum 10 cases $(25 \%)$ were treated by partial gastrectomy, and the rest 30 cases $(75 \%)$ were managed by palliative gastrojejunostomy. Truncal vagotomy and gastrojejunostomy was performed in 9 cases $(52.9 \%)$ and only gastrojejunostomy was done in 8 cases $(47.1 \%)$ of cicatricial duodenal ulcer. All patients of carcinoma of pancreas (7 cases) were managed by palliative gastrojejunostomy. One patient of GOO due to chemical ingestion was managed by gastrojejunostomy and one patient of gastric tuberculosis was managed by gastrojejunostomy followed by post operative ATT. Maximum case of antral carcinoma were managed by palliative gastrojejunostomy which is similar to the findings of Nayak (1984)0, Jaka et al (2013). Maximum cases of CDU were managed by truncal vagotomy and gastrojejunostomy.which is similar to the findings of Balint and Spence (1959), Kaushik and Moses (1973), Jaka et al (2013).
All cases of carcinoma of pancreas are managed by palliative gastrojejunostomy which is in accordance with Jaka et al (2013).

One case of gastric tuberculosis with GOO was treated by gastrojejunostomy followed by ATT with good result is in accordance with Nassir Alhaboob Arabi(2015), Jaka et al (2013).

Gastro-jejunostomy was the most frequent type of surgical procedure performed. This is in line with other studies done elsewhere. Kikuchi S (1999), Mittal A (2004) Alam TA (2003), Jaka et al (2013). The high rate of gastro-jejunostomy in this study is attributed to the large number of patients with malignant gastric outlet obstruction. Traditionally, malignant gastric outlet obstruction has been treated surgically, usually by creating a gastro-jejunostomy. More recently, the use of endoscopically placed self-expandable metal stents (SEMS) has become a routine practice Dongwook Oh, MD(2015), Miyazaki Y et al(2016) , Sasaki R et al (2016).However, this procedure was not popular in this study due to lack of this facility in the centre.

The presence of complications has an impact on the final outcome of patients presenting with gastric outlet obstruction. In the present study 17 patients $(25.75 \%)$ had 25 post operative complications. The commonest complication being surgical site infection (32\%) followed by post operative pyrexia (24\%), pneumonia (16\%), diarrhoea $(12 \%)$ paralytic ileus $(8 \%)$ and stomal obstruction $(8 \%)$.

In the present study, the postoperative complication rate was $25.72 \%$ and surgical site infection was the most common postoperative complications in the present study a figure which is In agreement with other studies. Jaka et al (2013).

The study shows pre operative fall in sodium level in $22.73 \%$ of cases with a mean of $132.13 \mathrm{mmol} / \mathrm{l}$. During the post operative period all the patients had normal sodium concentration with a mean of $136.66 \mathrm{mmol} / \mathrm{l}$.

The incidence of hyponatremia is similar to that of Kreel and Ellis (1965), Ranka Kshitiz(2013), Jawad Ahmad (2011). 33.33\% of cases were 
hypokalemic with a mean potassium level of 2.77 $\mathrm{mmol} / \mathrm{l}$ prior to surgery. Postoperatively all the patients had normal potassium concentration with a mean of $4.2 \mathrm{mmol} / \mathrm{l}$. these are in accordance with Kreel and Ellis (1965), Ranka Kshitiz(2013) and is less than the findings of Hyman S (1952), Jaka et al (2013) . 48.48\% patients were under weight with the mean weight of $50.92 \mathrm{~kg}$ during admission. Post operatively the mean weight decreases to $50.88 \mathrm{~kg}$. This finding is similar to that of Moody et al (1962), Mullen et al (1978).

25 complications occurred in 17 patients, of those only 3 complications occurred in those patients with normal weight whereas 22 complications occurred in those with low weight for height at the time of admission. This is similar to the findings of Mullen et al (1979), Mayor (1980). By applying chi-square test the $\mathrm{p}$ value is found to be 0.0017 which is highly significant. That is there is more likely to have post operative complications in those patients who are pre operatively under weight.

In the present study serum albumin levels were low in $24.24 \%$ cases as compared to $75.76 \%$ with normal serum albumin level. The mean serum albumin in the pre operative patients was 3.58 gm\%.The total number of postoperative patients with low serum albumin was $28.79 \%$ and the mean serum albumin level in these patients was 3.5 gm\%. Post operatively $56.06 \%$ patients had decreased albumin level as compared to the pre operative level, whereas $6.06 \%$ patients had increased and $37.88 \%$ patients had unchanged serum albumin level.

Pre operative hypoalbuminemia in $24 \%$ cases which is similar to the fingings of Mullen et al (1979), Moody et al .this is low than the findings of Jaka et al (2013)

Postoperative fall is similar to the findings of Casten et al (1943), Donald A Redelmeier(2009), 9 out of 16 patients with hypoalbuminemia $(56.25 \%)$ had some sort of post operative complications, whereas 8 out of 42 patients with normal serum albumin level (19.04\%) had postoperative complications. The $\mathrm{p}$ value is found to be 0.0028 which is highly significant. That is the patients with preoperative hypoalbuminemia are more likely to have postoperative complications as compared to those with normal serum albumin level.

These findings are similar to those of Mullen et al (1979), Martin Hubner(2016), Gibbs J (1999)

\section{Summary}

Sixty six cases of gastric outlet obstruction were admitted to the surgery department of VSS Institute of Medical Science And Research, Burla, Sambalpur during the period of November 2014 to November 2016.

- Majority of patients in the study belonged to 50-60 year age group.

- Male : female - 5.6:1

- Maximum cases were from low socio economic status.

- More than $90 \%$ cases are non vegetarians

- Irrespective of cause of obstruction, vomiting was the most consistent symptom followed by pain in the upper abdomen

- Commonest cause of gastric outlet obstruction in the study found to be carcinoma of gastric antrum followed by cicatricial duodenal ulcer.

- The commonest surgery performed was gastrojejunostomy. Surgery for malignant cases of GOO was decided by the stage of the disease at which the patient presented. Most of the patients with malignant cause were managed by palliative gastrojejunostomy.

- Commonest complication in the series found to be surgical site infection followed by post operative pyrexia and pneumonia.

- About one fourth of cases had hyponatremia pre operatively, but post operatively no patient had hyponatremia.

- Nearly one third of the patients with gastric outlet obstruction were with hypokalemia but post operatively all the patients have normal serum potassium level.

- Nearly half of the patients were underweight as per their weight for height 
and there were significantly high post operative complications in those patients.

- Near about one fourth of the patients in the present study had hypoalbuminemia prior to surgery. Post operatively there was decrease in serum albumin level in more than half of the patients. Post operative complications were significantly high in those patients who had pre operative hypoalbuminemia.

\section{Conclusion}

Gastric outlet obstruction is a common surgical problem in our setting and poses diagnostic and therapeutic challenges. It is more common among males with malignant causes being more prevalent. Gastric antral cancer is the most common malignant cause whereas cicatricial duodenal ulcer is the most common benign cause of gastric outlet obstruction.

Majority of patients presented to the hospital late with advance stage of disease. This might be due to less health related awareness in this region.

Vomiting and succussion splash were the most common symptoms and signs respectively in a case of gastric outlet obstruction.

Gastrojejunostomy is the most common surgery performed. Surgical procedures for malignant causes depend on the stage of the disease, operability and respectability of the tumor.

Some of the patients had electrolyte imbalance in the preoperative period, but postoperatively it is corrected in all the patients. Serum albumin level and weight of most of the cases decrease postoperatively. The preoperative nutritional status such as serum albumin and weight of the patient are highly significant in accessing the postoperative complication.

The result of this study suggests that early diagnosis, aggressive resuscitation and early institution of surgical management are of paramount importance if morbidity and mortality associated with gastric outlet obstruction are to be avoided.

\section{References}

1. Jaka H, Mchembe MD, Rambau PF, Chalya PL: Gastric outlet obstruction at Bugando Medical Centre in Northwestern Tanzania: a prospective review of 184 cases. BMC Surg 2013; 13(41):1-8.

2. Ranka K, Jain R, Jhanwar A: Gastric outlet obstruction in adults: A prospective study in tertiary care hospital of Karnataka: J Pharm Biomed Sci: 2013; 32(32): 1282-1286.

3. Studley HO. Percentage of weight loss: a basic indicator of surgical risk in patients with chronic peptic ulcer. JAMA. 1936; 106:458-460.

4. Mullen J L, Gertner M H, Buzby G P. Implications of Malnutrition in the Surgical Patient. Arch Surg. 1979; 114(2):121-125.

5. Lans HS, Stein Jr. IF, Meyer KA, Electrolyte abnormalities in pyloric obstruction resulting from peptic ulcer or gastric carcinoma. Annals Surg 1952; 135(4): 441-453

6. Kaushik S P, Mose. Gastric outlet obstruction in adults. Ind j. Surg. 1976;89:491

7. Howe $\mathrm{C} \mathrm{T}$, Le quesne $\mathrm{L}$ P. Pyloric stenosis: The metabolic effects. Brit. J. Surg. 1964; 51: 923-932 